\title{
AIRS-Light Instrument Concept and Critical Technology Development
}

\author{
Kevin Maschhoff* \\ BAE SYSTEMS \\ IR Imaging Systems, 2 Forbes Road, Lexington, MA 02421-7306
}

\begin{abstract}
Understanding Earth's climate, atmospheric transport mechanisms, and the hydrologic cycle requires a precise knowledge of global atmospheric circulation, temperature profiles, and water vapor distribution. The accuracy of advanced sounders such as AIRS/AMSU/HSB on NASA's Aqua spacecraft can match radiosonde accuracy. It is essential to fold those capabilities fully into the NPOESS, enabling soundings of radiosonde accuracy, every 6 hours around the globe on an operational basis. However, the size, mass, power demands, and thermal characteristics of the Aqua sounding instrument suite cannot be accommodated on the NPOESS spacecraft.
\end{abstract}

AIRS-Light is an instrument concept, developed under the Instrument Incubator Program, which provides IR sounding performance identical to the AIRS instrument, but uses advances in HgCdTe FPA technology and pulse tube cooler technology, as well as design changes to dramatically reduce the size, mass, and power demand, allowing AIRS-Light to meet all NPOESS spacecraft interface requirements. The instrument concept includes substantial re-use of AIRS component designs, including the complex AIRS FPA, to reduce development risk and cost. The AIRS-Light Instrument Incubator program fostered the development of photovoltaic-mode $\mathrm{HgCdTe}$ detector array technology for the $13.5-15.4 \mu \mathrm{m}$ band covered by photoconductive-mode HgCdTe arrays in AIRS, achieved state of the art results in this band, and substantially reduced the development risk for this last new technology needed for AIRS-Light implementation.

A demonstration of a prototype 14.5-15.4 $\mu \mathrm{m}$ band IRFPA in a reduced heat-load dewar together with the IMAS pulse tube cryocooler is in progress.

This paper will include a summary of the AIRS-Light instrument concept and of the advances in very long wavelength PV HgCdTe FPA technology.

\section{INTRODUCTION}

AIRS-Light is an Instrument Incubator Program with two main objectives. The first is the development of an instrument concept which would provide spectro-radiometric performance equivalent to that of NASA's AIRS ${ }^{(1)}$, but which meets NPOESS spacecraft interface requirements for the Cross-Track IR Sounder (hereafter, $\mathrm{CnIS}$ ) ${ }^{(2)}$. The second is to develop and demonstrate PV HgCdTe IRFPA module technology for the critical spectral region from 13.6-15.4 $\mu \mathrm{m}$, and demonstrate this technology together with the latest generation of miniature pulse tube cryocoolers in a thermal environment which simulates space flight conditions. A successful demonstration for these technologies would mean that all of the technologies necessary for this advanced high performance temperature/humidity sounder had been demonstrated, either on AIRS, or on AIRSLight, providing substantial risk reduction in advance of full scale instrument development. This instrument approach is applicable to the NPOESS Preparatory Project mission, the NPOESS mission, and other applications where AIRS-class sounding performance is required.

\section{INSTRUMENT CONCEPT}

AIRS meets all of the sounding performance requirements established for it by the Interagency Sounder Working Group. However, the final instrument places substantial demands on the spacecraft bus which have made it unsuitable for direct carry-over into the NPOESS program as the operational vertical temperature humidity sounder (called the CrIS). These requirements, together with corresponding characteristics for AIRS and AIRS-Light are listed in Table 1. In practice, these requirements are closely interrelated. The requirement for NPOESS instruments to conduct at most $3 \mathrm{~W}$ of power to the spacecraft essentially requires the instrument to dissipate its waste heat to space. The size requirements limit the area available for radiating the instrument waste heat to space. This combination of size and thermal limits on the spacecraft interface are the dominant interface requirements for CrIS, and AIRS-Light.

* Correspondence: Email: kevin.maschhoff@baesystems.com Telephone: 781-863-3900 
Table 1: Sounding Instrument Interface Requirements and Characteristics

\begin{tabular}{|l|l|l|l|}
\hline Interface Requirement & AIRS & AIRS-Light Estimate & CrIS ICD \\
\hline Size $(\mathrm{cm})$ & $140 \times 78 \times 76 \mathrm{~cm}$ & $60 \times 45 \times 40 \mathrm{~cm}$ & $60 \times 40 \times 40 \mathrm{~cm}$ \\
\hline Mass & $176 \mathrm{~kg}$ & $<68 \mathrm{~kg}$ & $<77 \mathrm{~kg}$ \\
\hline Power & $224 \mathrm{~W}$ & $<70 \mathrm{~W}$ & $<85 \mathrm{~W}$ \\
\hline $\begin{array}{l}\text { Thermal Interface with } \\
\text { Spacecraft }\end{array}$ & Spacecraft cooling required & $\begin{array}{l}\text { Thermally independent } \\
\text { of spacecraft }\end{array}$ & $\begin{array}{l}\text { Thermally independent of } \\
\text { spacecraft }\end{array}$ \\
\hline
\end{tabular}

The AIRS-Light instrument performance requirements were taken directly from the AIRS instrument requirements as captured in the AIRS Functional Requirements Document. Table 2 shows the critical subset of these requirements which drive instrument design.

Table 2: Selected AIRS and AIRS-Light Functional Requirements

\begin{tabular}{|c|c|c|c|c|}
\hline Paragraph \# & Parameter & Requirement & Units & Comments \\
\hline 3.1 .2 & Scan Period & $8 / 3$ & sec & \\
\hline 3.1 .2 & Scan Field & $+/-49.5$ & deg & \\
\hline \multirow[t]{3}{*}{3.1 .3} & Spatial Response & $<1.1$ & deg & FWHM at nadir \\
\hline & & $<2.5$ & $\operatorname{deg}$ & $>99 \%$ of integrated response \\
\hline & & $<7.5$ & deg & $>99.95 \%$ of integrated response \\
\hline 3.1 .3 .1 & $\begin{array}{l}\text { Measurement } \\
\text { Simultaneity }\end{array}$ & $>0.99$ & & $\begin{array}{l}\text { includes boresight alignment, spatial response } \\
\text { nonuniformity, and timing errors }\end{array}$ \\
\hline 3.1 .4 .1 .1 & Spectral Coverage & $\begin{array}{l}3.74-4.61 \\
6.20-8.22 \\
8.80-15.4\end{array}$ & $\mu \mathrm{m}$ & \\
\hline 3.1 .4 .1 .2 & $\begin{array}{l}\text { Spectral } \\
\text { Resolution }\end{array}$ & $\begin{array}{l}900-1400 \\
1200+1-60\end{array}$ & $\lambda / \Delta \lambda$ & at $14 \mu \mathrm{m}$ \\
\hline 3.1 .4 .1 .5 & Spectral Stability & $<5$ & $\%$ & of $\Delta \lambda$ in any $24 \mathrm{hr}$ period \\
\hline 3.1 .4 .1 .6 & $\begin{array}{l}\text { Spectral } \\
\text { Knowledge }\end{array}$ & $<1$ & $\%$ & of $\Delta \lambda$ \\
\hline 3.1 .5 .1 & Sensitivity & $\begin{array}{l}<0.2(3.74-13.4) \\
<0.14(\text { near } 4.2) \\
<0.35(>13.4)\end{array}$ & $\mathrm{K}$ & for a resolution element, for a $250 \mathrm{~K}$ scene \\
\hline 3.1 .7 .1 & $\begin{array}{l}\text { Radiometric } \\
\text { Accuracy }\end{array}$ & $\begin{array}{l}<3 \\
\text { or } 4 / \mathrm{SNR}\end{array}$ & $\%$ & over full dynamic range \\
\hline 3.1 .9 .1 & Polarization & $<25 \%$ & $\%$ & $<5 \mu \mathrm{m}$ \\
\hline
\end{tabular}

The instrument design approach, developed to meet these challenging interface requirements while preserving AIRS-class sounding performance, has the following major elements:

- Dramatic reduction in detector cooling power requirements using new technology

- Dramatic reduction in spectrometer size and mass

- Substantial preservation of AIRS heritage in critical components.

The AIRS-Light instrument approach meets the challenging NPOESS interface requirements. The concept is shown in Figure 1. Major aspects of this instrument concept are described below. 


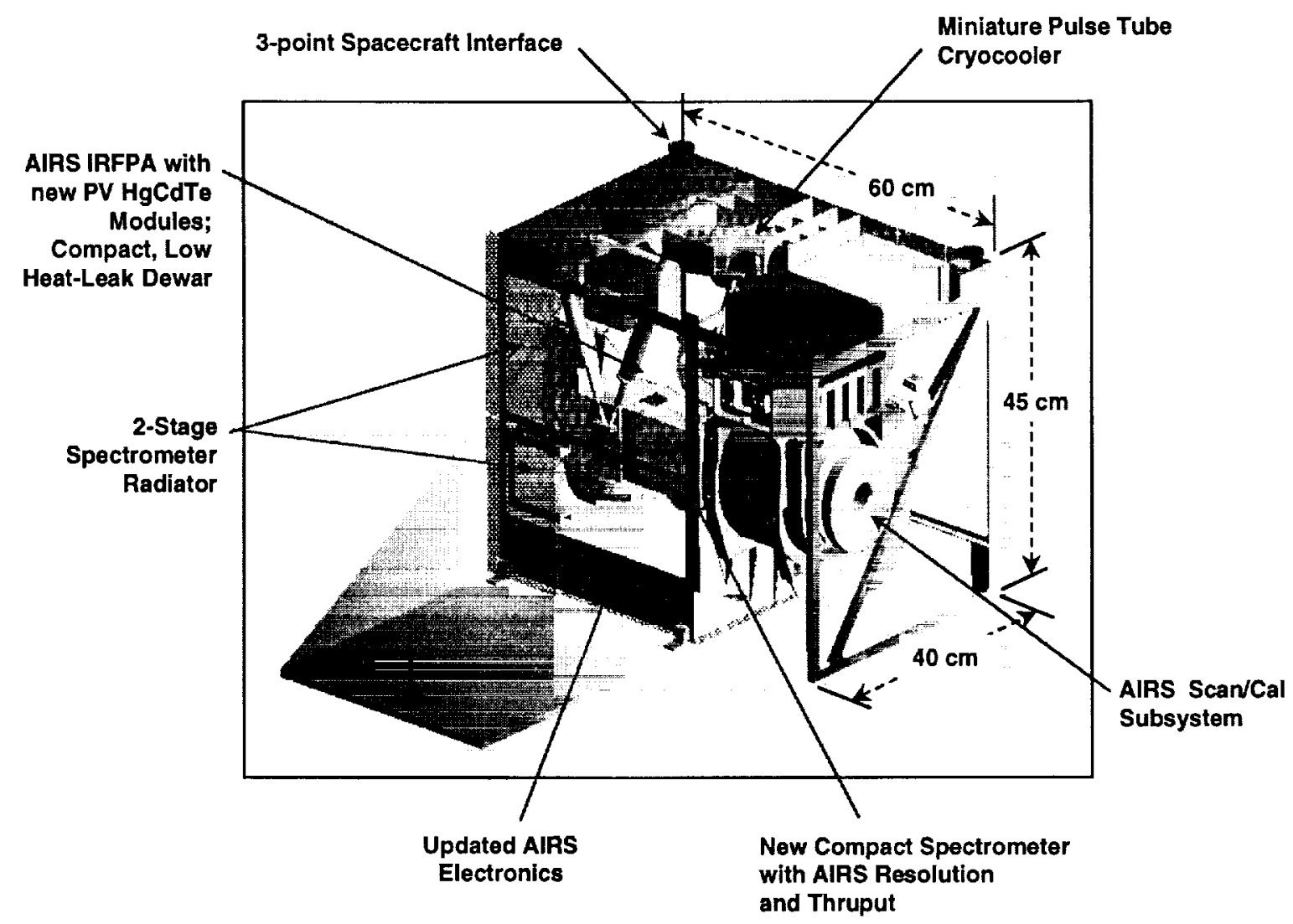

Figure 1. AIRS-Light Instrument Concept

The most significant change in instrument design approach when moving from AIRS to AIRS-Light is the extension of the spectral coverage by photovoltaic mode $\mathrm{HgCdTe}$ detectors from $13.4 \mu \mathrm{m}$ (for AIRS) ${ }^{(3)}$ to $15.4 \mu \mathrm{m}$ (for AIRS-Light). This change allowed a substantial reduction in the number of electrical leads from the Detector/Dewar to the warm electronics (from $\sim 600$ in AIRS to $\sim 200$ in AIRS-Light), which reduces the parasitic thermal load on the cooler. The AIRS-Light FPA then would use $10 \mathrm{PV} \mathrm{HgCdTe} \mathrm{IRFPA} \mathrm{modules} \mathrm{taken} \mathrm{directly} \mathrm{from} \mathrm{the} \mathrm{AIRS} \mathrm{design,} \mathrm{and} \mathrm{two} \mathrm{new} \mathrm{ones} \mathrm{covering} \mathrm{the} \mathrm{13.4-}$ $15.4 \mathrm{~mm}$ band which directly replace the two PC modules which covered this band for AIRS.

A second critical change is to use one pulse tube cryocooler, (still using redundant cooler control electronics). This change leads to substantial reduction in parasitic thermal load also. The AIRS instrument has two redundant pulse tube coolers, and the off-load of the redundant cooler represents nearly $400 \mathrm{~mW}$ of the total heat load. Total heat load for the AIRS-Light Detector/Dewar is estimated at between 500 and $600 \mathrm{~mW}$, less than $1 / 3^{\text {rd }}$ of the AIRS heat load, and small enough to be absorbed by a single miniature pulse tube cryocooler.

The switch to using PV HgCdTe for all IR channels has a dramatic impact on the IR spectrometer as well. The AIRS spectrometer ${ }^{(1)}$ has a multi-aperture pupil imaging design. The use of photoconductive mode HgCdTe for the band from 13.4 to $15.4 \mu \mathrm{m}$ required that one of the 11 apertures on AIRS be optically chopped, since the PC devices must be AC coupled in this application, but optical chopping all channels would have placed unacceptable demands on sensitivity in other spectral regions. The chopping requirement substantially constrained the allowed optical design solution space for AIRS. The AIRSLight spectrometer does not have this constraint, and the resulting spectrometer can be physically smaller. The spectrometer was constrained to interface directly with the existing 12 -Module AIRS FPA ${ }^{(3)}$ in order to maximally utilize the NASA investment in AIRS and minimize development risk for AIRS-Light by avoiding unnecessary IRFPA module redesign. The spectrometer is less than $1 / 4^{\text {th }}$ the size of the AIRS spectrometer. It has an estimated mass of less than $10 \mathrm{~kg}$, which is less than $1 / 3^{\text {rd }}$ that of AIRS.

The AIRS spectrometer is cooled to $150 \mathrm{~K}$ to reduce instrument self-radiation in the spectral regions beyond $7 \mu \mathrm{m}$. The spectrometer is passively cooled by a 2-stage radiator on the anti-sun instrument face, with a deployable earth shield to prevent earthshine from striking the coldest stage. This radiator and shield are the components of AIRS with the largest size- the same function must be accomplished in AIRS light in a much smaller envelope. The substantial reduction in 
spectrometer mass and size allow a substantial reduction in the size of the passive radiator, since the load on the radiator (conductive and radiative loads) is much smaller. In addition, AIRS-Light will employ Linear Variable Filters in place of broad band order-sorting filters ${ }^{(1)}$ for the longer spectral bands. This will allow the AIRS-Light spectrometer to be held at a temperature of $\sim 185 \mathrm{~K}$ rather than the $150 \mathrm{~K}$ of AIRS. With these changes, the passive radiator and its shield now fit within the CrIS instrument size envelope.

The AIRS-Light electronics provide essentially the same functions as the AIRS electronics. Selected changes must be made in the design to replace components which would be obsolete. The most siginficant change of this type is the instrument control processor, which would be replaced by a RAD 750. The most significant change for the electronics is geometrical. Electronics for AIRS are placed in two card cage assemblies which are close to the instrument/spacecraft interface, and are cooled by the spacecraft. In AIRS-Light, the electronics are spatially reconfigured and placed on the earth-facing panel of the instrument to facilitate the radiation of their waste heat to space through a nadir-face radiator.

\section{VLWIR PV HgCdTe IRPFA MODULE DEVELOPMENT}

The preceding discussion highlights the importance of the extension of $\mathrm{PV} \mathrm{HgCdTe}$ technology to longer wavelengths as a necessary development to support the AIRS-Light instrument concept and related NASA instrument efforts such as GIFTS and related NPOESS efforts such as CrIS. To retire the risk of this development, the AIRS-Light program funded a demonstration of the PV detector technology in this spectral region. The demonstration included the growth and fabrication of two lots of PV HgCdTe wafers, wafer-level evaluation using test arrays, and the build and test of a PV HgCdTe FPA module. The PV FPA module is a hybrid assembly which unites an HgCdTe detector array with a CMOS readout integrated circuit (ROIC). For the purposes of this demonstration, the selected array was assembled with an AIRS M10 ROIC which is similar, but not identical to an ROIC one would design for an AIRS-Light module for the 13.4 to $15.4 \mu \mathrm{m}$ spectral region (covered by M11 and M12 for AIRS). However, it has sufficient charge capacity and other characteristics which enable a suitable demonstration of the VLWIR HgCdTe.

Figure 2 shows an electron micrograph of one of the $\mathrm{HgCdTe}$ wafers processed for AIRS-Light. The wafer contains test arrays as well as long rows of detectors (bi-linear) which are diced out for use as arrays for the IRFPA module. The wafers were grown via BAE-Systems' two-layer $\mathrm{P} / \mathrm{n}$ liquid phase epitaxy process. This process had recently been upgraded to substantially reduce the frequency of graphite particle contamination. Baselayer doping and cap-layer thickness are carefully controlled to avoid heterojunction barrier formation. Wet chemical etching is used to define detector active areas. Detector mesa sidewalls are passivated with CdTe deposited at low temperature in an MBE reactor. Photolithographic definition was performed using a projection mask aligner to avoid any contact damage. This processing was especially tailored to the very delicate characteristics of VLWIR HgCdTe, which is very easily damaged by mechanical or thermal stresses. The results, presented below, show successful fabrication of this delicate material into high performance detector arrays with spectral cutoff wavelengths beyond $16 \mu \mathrm{m}$ at $60 \mathrm{~K}$.

Figure 3 shows detector impedance-area product data for a collection of photodiodes which replicate those in the IRFPA module. The data are from the best performing wafer, designated LN729, and were taken at a detector temperature of $60 \mathrm{~K}$ the target operating temperature for an AIRS-Light FPA. These data, with average zero bias impedance-area product of 15.6 $\Omega-\mathrm{cm}^{2}$ meet the AIRS-Light detector flowdown requirements, which call for $R_{0} A$ between 15 and $20 \Omega-\mathrm{cm}^{2}$ for the 13.4$15.4 \mu \mathrm{m}$ spectral band FPA modules, and are approximately $1 / 3^{\text {rd }}$ of the theoretical diffusion current limit for this alloy composition and doping density. Figure 4 shows detector quantum efficiency for the same set of detectors. Average measured quantum efficiency of approximately $60 \%$ is excellent for these arrays which have no antireflection coating for this demonstration and implies an internal diode quantum efficiency of $77 \%$. Application of a low-loss A/R coating was intentionally deferred to a flight instrument development program. Spectral response measurements, depicted for one of these photodiodes in Figure 5, show a spectral cutoff near $16.37 \mu \mathrm{m}$ at $60 \mathrm{~K}$, and high response in the 13.4-15.4 $\mu \mathrm{m}$ region. Preliminary measurements indicate that $1 / \mathrm{f}$ noise for the majority of phododiodes is low, and meet the flowdown requirement for the Tobin $\alpha_{\text {-dark }}$ coefficient of $<1 \times 10^{-4}$ Amps/Amp. Finally, Figure 6 shows dark current data taken from and IRPFA module assembled with an array from wafer LN729. The data are taken at a temperature of $57 \mathrm{~K}$ and a nominal reverse bias of $5 \mathrm{mV}$. Of the 150 diodes on the A-side of the IRPFA module, 137 (more than 90\%) show dark current levels less than $2 \mathrm{x}$ the average of $10 \mathrm{nA}$, indicating a high level of diode operability for this spectral cutoff. The AIRS-Light instrument concept calls for two or more rows of detectors in this band, providing each spectral sample with multiple detectors. This strategy, together with $85-90 \%$ operability indicated in Figure 6 meet the $98 \%$ operability required at the spectral sample level. 

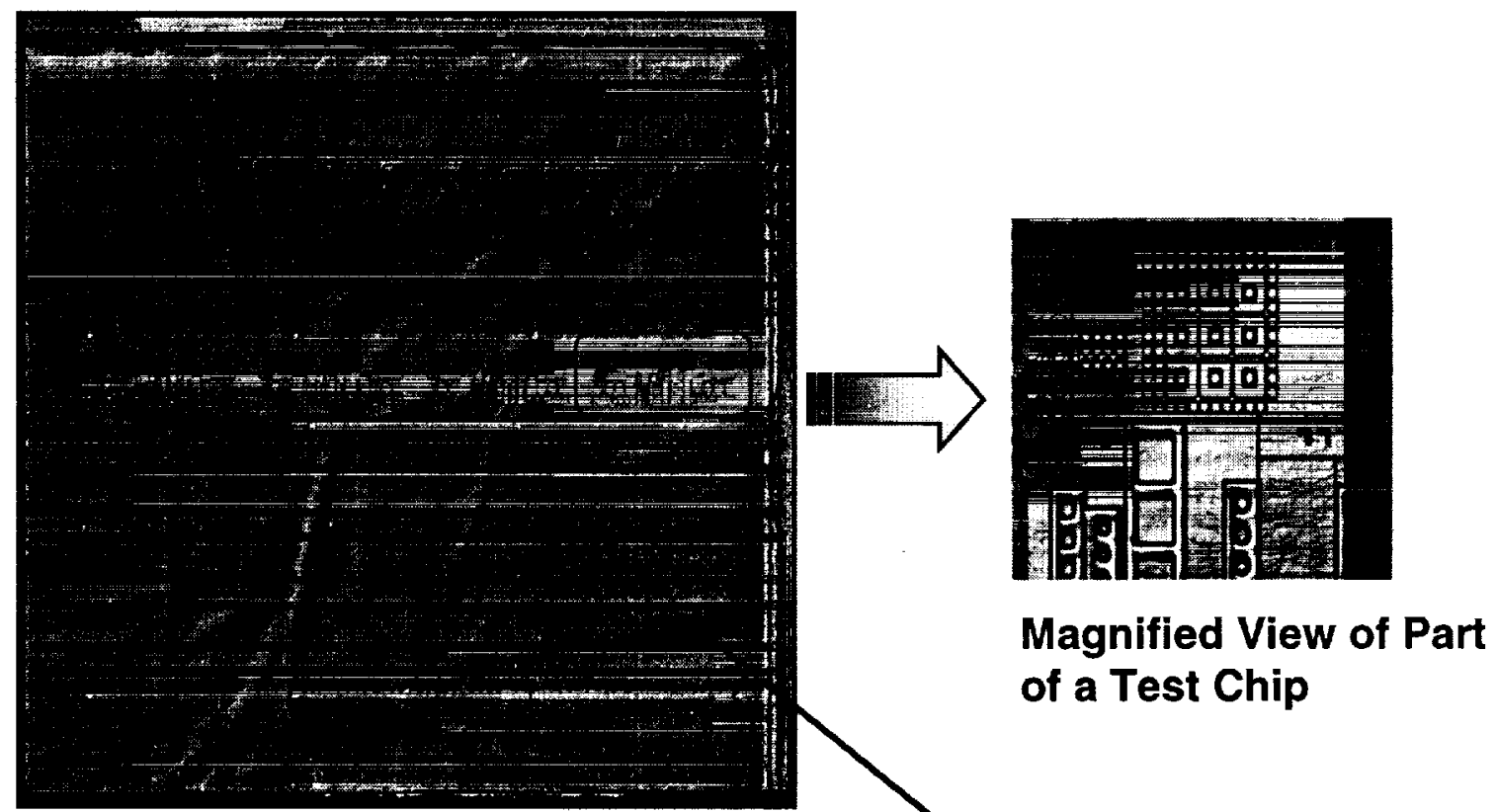

Continuous Detector Array Strips

Figure 2. Wafer LN719 in Process Showing Patterned Arrays

\section{LN729 RA product at $60 \mathrm{~K}$}

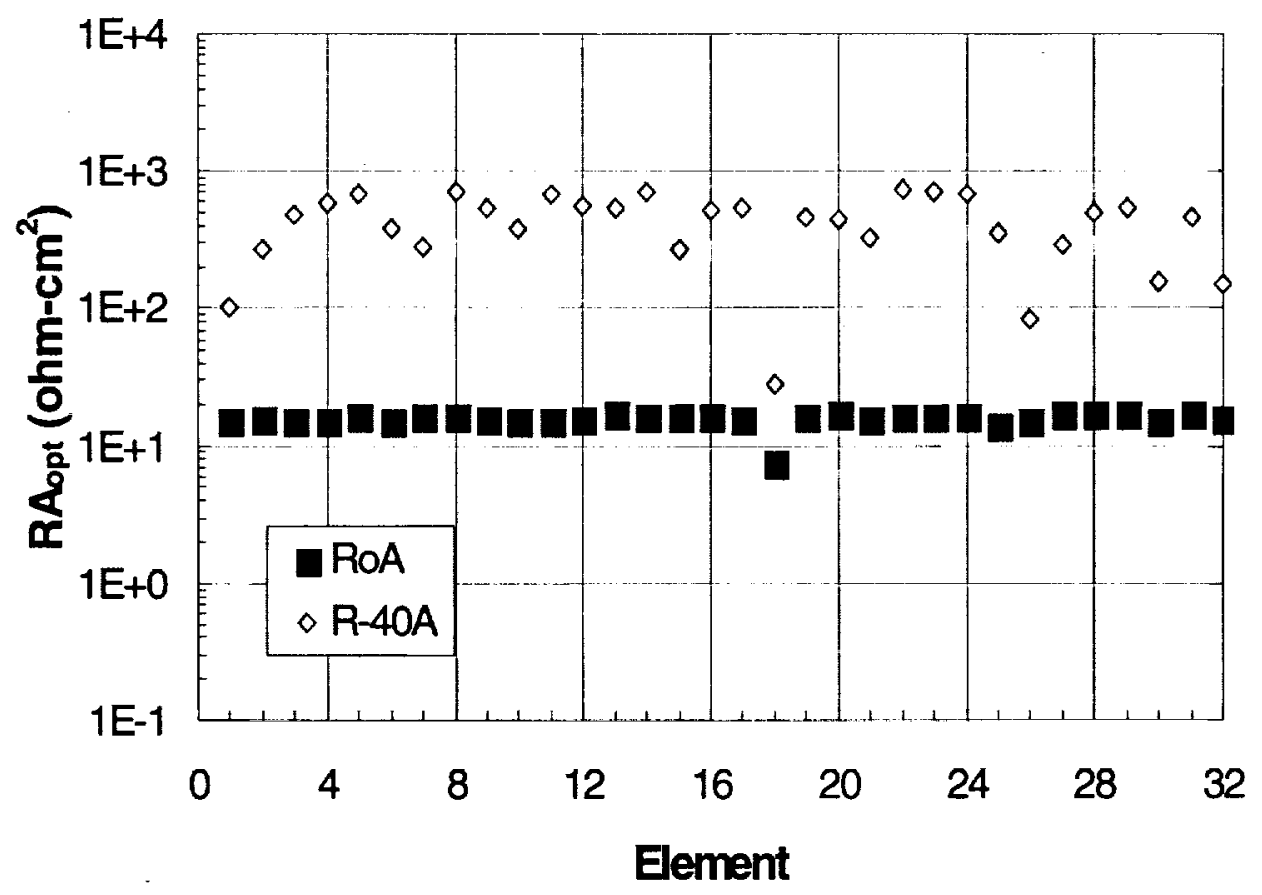

32- Element minl-array of $50 \times 100 \mu \mathrm{m}$ pixels

Figure 3. Impedance-area Product for HgCdTe Photodiodes with Spectral Cutoff Near $16.4 \mu \mathrm{m}$ at $60 \mathrm{~K}$ 


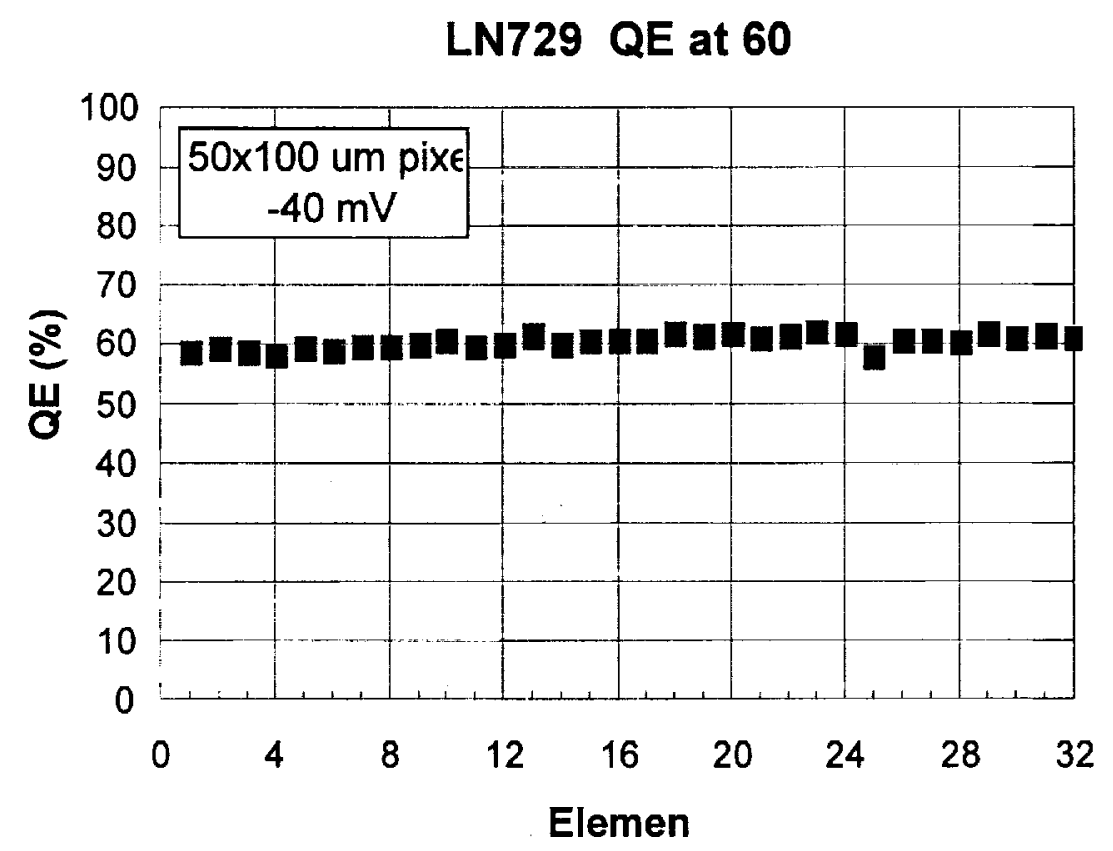

32- Element minl-array . No Antireflection Coating

Figure 4. Excellent Uniformity of Quantum Efficiency

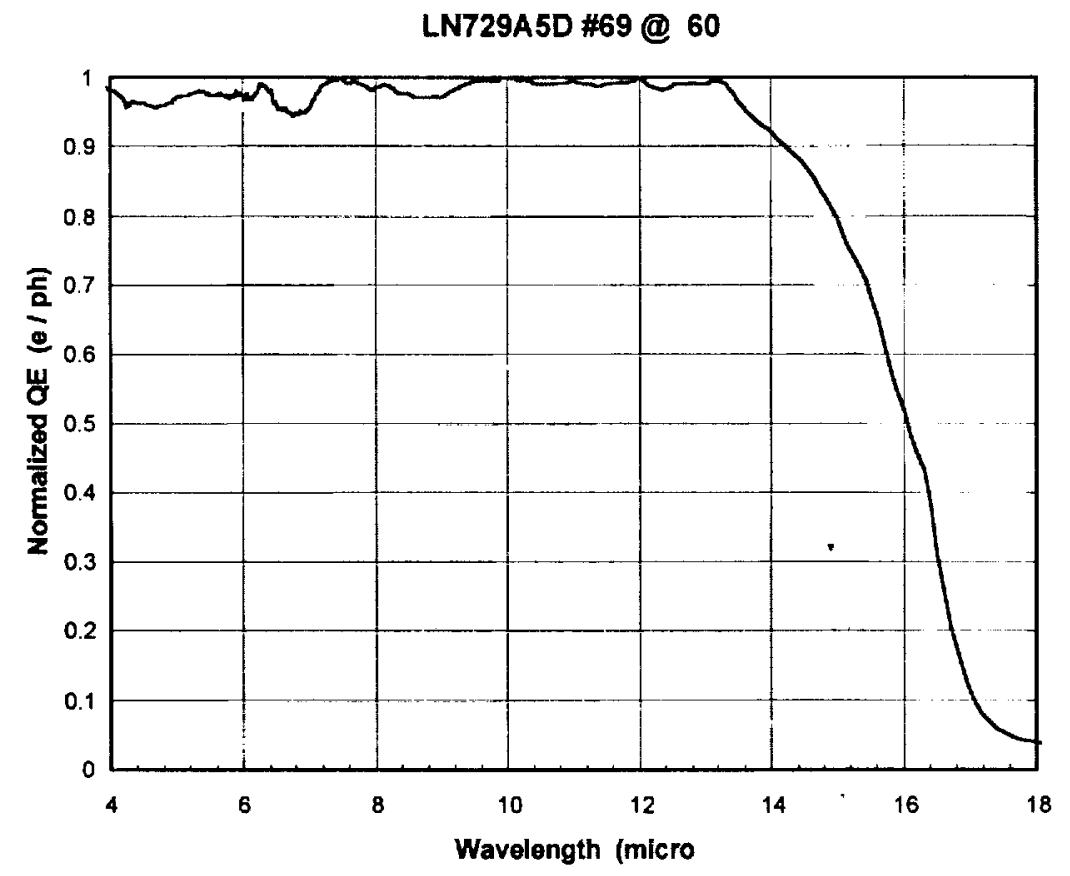

Cutoff $=16.37$, Meets M12 Goal of $16.17-16.94 \mu \mathrm{m}$

Figure 5. Classical Spectral Response with M12 Band Cutoff 
While meeting the AIRS NE $\Delta$ T requirements, shown here.

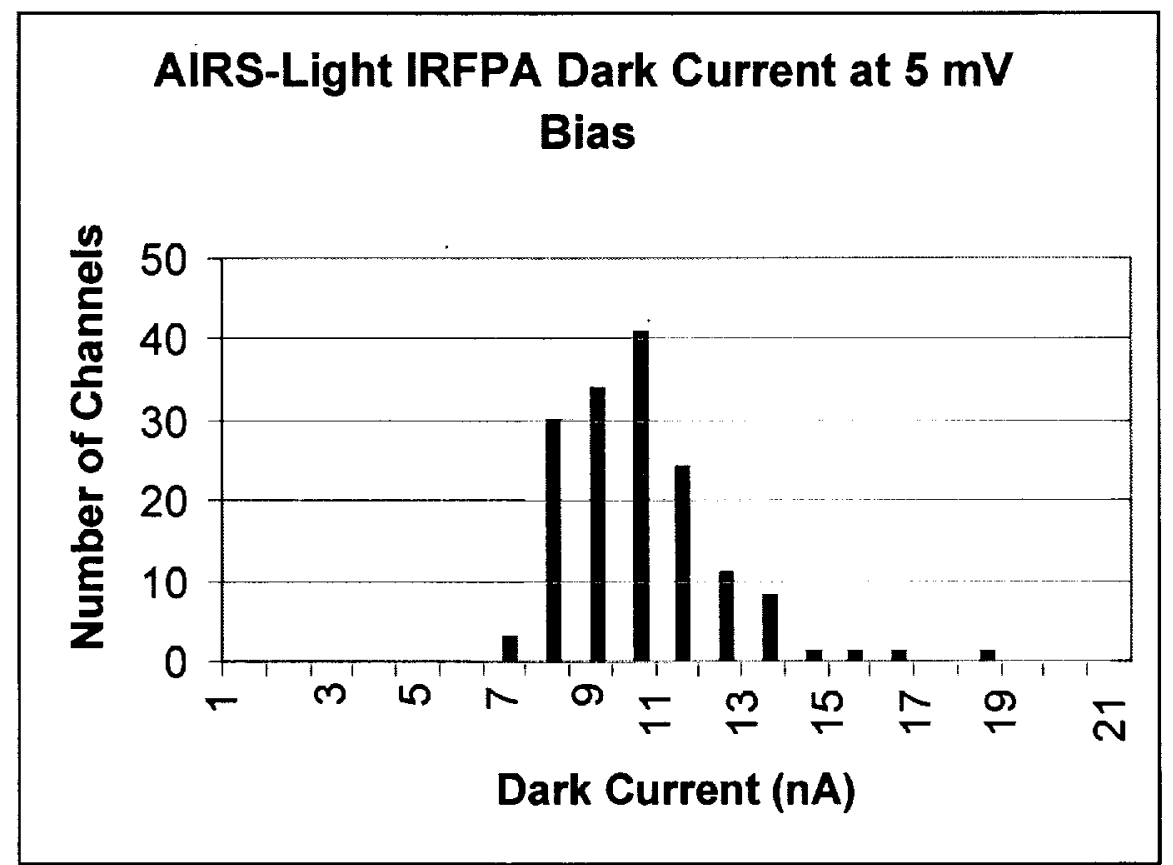

Figure 6. Dark Current for a PV HgCdTe Array with $16.4 \mathrm{~mm}$ Cutoff Wavelength Measured through an AIRS M10 ROIC Module is Shown for an FPA Temperature of $57 \mathrm{~K}$. Data for 137 Diodes are Included in the Figure. Only 13 Diodes Show Excess Leakage Current.

\section{INSTRUMENT PERFORMANCE PROJECTION AND SUMMARY}

The radiometric performance model developed and validated during the AIRS program was applied to AIRS-Light for a variety of spectrometer temperatures. Model NEDT vs spectral channel wavelength is shown in Figure 7 for a variety of spectrometer temperatures, along with the AIRS NEDT requirements. Performance for channels below $13.4 \mu \mathrm{m}$ is projected from the AIRS as-built performance data for the higher spectrometer temperatures. Noise model estimates based on detector characteristics shown in this paper are used for the upper two micrometers of spectral coverage. These projections, based on a validated instrument model and demonstrated detector results, show that AIRS-Light would meet the AIRS instrument requirements with acceptable margin. Spectral resolution characteristics of AIRS-Light would be essentially identical to the highly locallized spectral response characteristics demonstrated on AIRS. Similarly, the high degree of spatial coregistration of the AIRS spectral channels would be carried over to AIRS-Light due to the similarity of the optical designs. In short, the AIRS-Light instrument would meet the NPOESS requirements for temperature and humidity profile environmental data records.

\section{SUMMARY}

The AIRS-Light Incubator Program has substantially reduced risk for instrument development by demonstrating the key PV VLWIR HgCdTe technology. This, together with the advent of miniature versions of the pulse tube cryocooler mean that no new technology development is required for produce AIRS-Light. The AIRS-Light instrument study has demonstrated that the accommodation requirements of size, mass, power, and thermal independence established for CrIS can be met by AIRSLight at low risk. The final stage of the AIRS-Light Instrument Incubator program is in progress. In this phase, the AIRSLight prototype IRPFA module will be integrated within a lower heat-load version of the AIRS Dewar. This assembly will be integrated with a miniature pulse tube cooler produced by TRW, and placed within a thermal/vacuum chamber in the AIRS Calibration and Test Facility in Lexington, MA. This configuration will allow the VLWIR radiometric performance of AIRS-Light to be verified within a thermal environment that closely simulates the space flight environment, and will allow a refinement of the required operating temperature of the IRFPA, and the anticipated thermal loads on the cooler. 


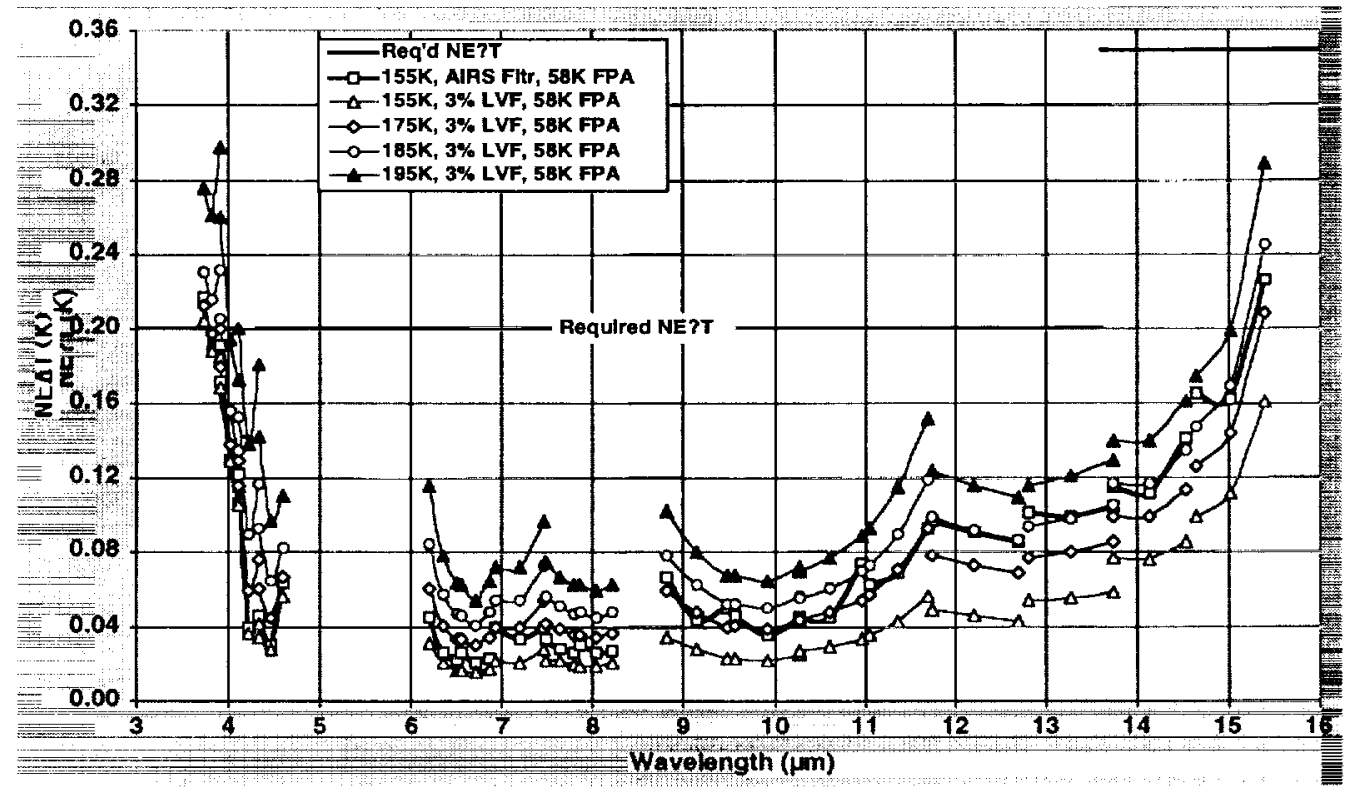

* Based on AIRS As-Built Data

Figure 7. Use of Linear Variable Filter Allows Higher Spectrometer Operating Temperature $-185 \mathrm{~K}^{\star}$

\section{ACKNOWLEDGEMENTS}

The work was funded by NASA's Instrument Incubator Program under the oversight of Mr. Pepper Hartley. The support of NASA and Mr. Hartley are gratefully acknowledged.

\section{REFERENCES}

1. Morse, P.G., Bates, J.C., Miller, C.R. etal, (1999) Proc. SPIE Vol. 3870, p. 281

2. National Polar Environmental Satellite System (NPOESS) requirements can be funded on the Integrated Program Office web site, www.ipo.noaa.gov

3. Rutter, J., Libonate, G.S., Robillard, G. etal, (1998) Proc. SPIE Vol. 3437, p. 249 